\title{
La higiene en la ciudad de Morelia, México, durante el régimen del porfiriato
}

\author{
Hygiene in the city of Morelia, Mexico, during the Porfiriato regime
}

Oscar A. Sánchez-Pérez y Alain R. Rodríguez-Orozco*

Facultad de Ciencias Médicas y Biológicas Dr. Ignacio Chávez, Universidad Michoacana de San Nicolás de Hidalgo, Morelia, Michoacán, México

\section{Resumen}

Este trabajo se realizó con el objetivo de documentar el estado de la higiene y los preceptos que a ella atañen en la ciudad de Morelia, México, durante el porfiriato. Para ello se consultaron documentos de la época, como periódicos, memorias de gobierno, leyes y reformas del Bando de Policía decretados por los gobiernos en turno en materia de higiene privada y pública.

Palabras clave: Higiene. Historia de la medicina. Morelia. Siglo XIX. Porfiriato.

\begin{abstract}
This work was carried out with the objective of documenting the state of hygiene and the precepts that concern it, in the city of Morelia, Mexico during the Porfiriato period. In order to do that, documents of that time were consulted such as newspapers, government reports, laws and reforms of the Police Band decreed by the governments, concerning public and private hygiene.
\end{abstract}

Key words: Hygiene. History of medicine. Morelia city. $19^{\text {th }}$ century. Porfiriato.

\section{Introducción}

Una de las contribuciones en materia médica más importantes del último tercio del siglo XIX fue el proceso que llevó a la transformación de la higiene pública y privada. En el tiempo y el espacio en que se delimita el tema de esta investigación, «el porfiriato", se encuentran involucradas dos teorías de transmisión de la enfermedad. En el inicio del porfiriato, para la explicación de la transmisión de las enfermedades se usó la teoría miasmática del contagio, y bajo sus principios médicos operaba la salud pública. Durante la segunda mitad del siglo XIX se vivió en el mundo un cambio de paradigma referente al origen de las enfermedades. Los descubrimientos que se dieron en la microbiología demostraban de manera convincente la existencia de seres invisibles para el ojo humano que estaban presentes en el medio ambiente y que habían estado presentes en toda la historia de la Humanidad causando enfermedades, las cuales habían sido explicadas de muchas formas. Sin embargo, no

\section{Correspondencia:}

*Alain R. Rodríguez-Orozco

Rafael Carrillo esquina Salvador González Herrejón s/n

Bosque Cuauhtémoc, Col. Centro

Fecha de recepción: 02-09-2020

Fecha de aceptación: 27-10-2020

E-mail: alain.rodriguez@umich.mx 
todos los especialistas en el estudio de las causas de las enfermedades aceptaban estas ideas, por lo que surgieron varios debates en torno a esta nueva teoría'. Para inicios del siglo XX, la teoría del germen había sido prácticamente aceptada gracias a numerosas publicaciones médicas.

Otro punto a considerar para el desarrollo de la higiene en Morelia durante el porfiriato fue el gradual reconocimiento de esta como ciencia, ya que de la mano de las ideas europeas, especialmente las francesas, la higiene rebasó sus intereses filantrópicos y adquirió un papel científico y político que predominaría durante las últimas décadas del siglo $\mathrm{XIX}^{2}$.

Este trabajo se realizó con el objetivo de documentar el estado de la higiene y los preceptos que a ella atañen en la ciudad de Morelia, México, durante el porfiriato.

\section{Panorama de la higiene en Morelia durante el porfiriato}

Para el año 1877, la higiene de la ciudad de Morelia presentaba una seria crisis administrativa. La carencia de fondos municipales se reflejó en ramos tales como el alumbrado público, el aseo de las poblaciones y el abastecimiento de aguas potables. La salubridad pública resintió ese abandono y se documentaron alarmantes cifras de mortalidad en algunas poblaciones del Estado, y era común la apatía de los ayuntamientos de Morelia en cuanto a la implementación de medidas para la salubridad ${ }^{3}$.

En lo que respecta a la obligación del Estado, la falta de recursos o la mala administración de estos fueron motivos por los cuales la salubridad se encontraba tan deficiente. En el mes de marzo del año 1877 se presentaron en la ciudad algunos casos de tifo y de fiebre tifoidea. Su número aumentó hasta considerarse el peligro de una epidemia en caso de que no se tomasen las medidas necesarias. La tala masiva de árboles no solo produjo una falta de agua debido a la escasez de lluvias, sino que también afectó la calidad del aire. Con la desaparición de los bosques, la población se consideraba más desprotegida, ya que se creía que los bosques eran las barreras que la naturaleza oponía a la extensión y la propagación de los aires viciados que, llevando consigo los miasmas pestilentes y epidémicos, difundían la desolación y la muerte ${ }^{3}$.

Podemos observar en los dos párrafos anteriores la relación que había con la teoría miasmática de la enfermedad. Uno de los principales elementos que se destacan en los escritos sobre la salubridad y la higiene de esa época era el aire, principal conductor de los miasmas pútridos que viajaban desde sus principales focos de infección hacia los organismos saludables.

Los desmontes no eran las únicas observaciones que se hacían a la salubridad de la ciudad; a esto se agregaban las malas condiciones del acueducto por donde pasaba el agua que se consumía para satisfacer las necesidades de la ciudad, así como la mala construcción de los panteones que estaban rodeados de muros muy pequeños sin cumplir con las normas de higiene de la época, por lo que hacían de ellos un espacio potencialmente abierto para las enfermedades miasmáticas y virulentas ${ }^{3}$.

También se incluían, dentro del paisaje infeccioso que se describía en los reportes de las memorias de gobierno, los pantanos. Estos fueron una constante preocupación durante las últimas décadas del siglo XIX. Se formaban al norte, al poniente y un poco al sur de la ciudad de Morelia durante la época de Iluvias. Una de las principales causas de su formación era la falta de atención que se daba a la conservación y limpieza periódica del Río Grande ${ }^{3}$.

La tala excesiva de árboles y la formación de pantanos coexistían junto a otras situaciones señaladas por la Junta de Salubridad en 1877, como el desaseo de la población, los basureros al aire libre en varias partes de la ciudad, la falta de agua en las fuentes públicas y la poca inspección que se daba a establecimientos públicos como tocinerías, casas de abasto y expendios de carne; las zahúrdas eran focos de descomposición dentro de la ciudad ${ }^{3}$. Estos eran algunos de los principales problemas de higiene que presentaba la salubridad en Morelia a inicios del porfiriato, y los primeros en que se trabajaría para resolverlos y conseguir mejorar la higiene de los morelianos.

Entre los años 1878 y 1887 se publicaron en la prensa moreliana varias noticias sobre las mejoras realizadas en aspectos vinculados a la salud. En algunos números del Periódico Oficial del Gobierno del Estado de Michoacán de Ocampo encontramos noticias y artículos referentes a la importancia de los bosques en la salud pública ${ }^{4}$, la prevención de males en el consumo de carne ${ }^{5}$, el abastecimiento de agua ${ }^{6}$, la inauguración de baños públicos ${ }^{7}$, la construcción del nuevo panteón ${ }^{8}$ y la desecación de pantanos en la ciudad'.

La desecación de los pantanos continuaba siendo una de las principales prioridades para las autoridades de la ciudad. Las obras que se realizarían para 
terminar de manera permanente con la formación de pantanos incluyeron la ampliación del cauce y el levantamiento del margen del Río Grande, ya que con el paso de los años había sido modificado por algunos particulares con el afán de ensanchar sus propiedades, y habían estrechado el río ocasionando que este se tapara con el escombro que arrastra y terminara desbordándose y dando origen a los tan temidos focos de infección que eran los pantanos ${ }^{10}$.

A la ardua tarea de la desecación de los pantanos se fueron sumando algunas otras disposiciones de salubridad, relativas al aseo y la limpieza de la ciudad, al establecimiento del sistema de atarjeas, mejoras en el panteón municipal, la inauguración de baños, la desinfección de pozos y las reformas que se realizaron al Bando de Policía.

En el número de la Gaceta Oficial correspondiente al 17 de abril de 1887 se publicaron las adiciones y reformas al Bando de Policía vigente en la Municipalidad:

«Art. $1 .^{\circ}$. Todas las casas, portales y edificios públicos - excepto los de pañería- que estén sucios ó maltratados, así como las tapias de las huertas, solares y jardines situados en los paseos públicos ó que miren á calles muy transitadas, se enjarrarán y pintarán convenientemente en todos los lados que tengan á la vía pública, por cuenta de sus respectivos dueños ó poseedores, en el improrrogable término de un mes contando desde la publicación de estas disposiciones, bajo el concepto de que la infracción de este artículo se castigará con la multa de uno á veinticinco pesos, sin perjuicio de que el Ayuntamiento mande pintar la finca á costa del dueño ó poseedor.

Art. $2^{\circ}$. La disposición á que se refiere el artículo anterior, será obligatoria cada dos años, fijándose para lo sucesivo al efecto, los meses de Octubre, Noviembre y Diciembre de los años correspondientes.

Art. $3 .^{\circ}$. Se reforma el artículo 38 del Bando de Policia, en Términos siguientes "Diariamente y á los ocho de la mañana estarán regadas con agua limpia y barridas todas las calles de la ciudad y aseados los caños; teniendo esta obligación todo vecino, sin excepción alguna, en la extensión del frente y lados de su casa, aun cuando cerca no hubiere habitaciones; siendo extensiva dicha obligación para los encargados de las Iglesias, Hospitales, Hospicios, Cárceles, Cuarteles, Colegios y edificios públicos; para los dueños de casas desocupadas, para los aguadores, respecto de las plazas y plazuelas donde existen fuentes públicas, y que estén inscritos en ellas; y por último, para los vendedores, tratándose de las plazas, mercados ó puntos donde se sitúen antes de levantar su puesto ó vendimia. Los infractores de este artículo serán castigados con una multa que no baje de veinticinco centavos ni exceda de veinticinco pesos, á juicio de los munícipes encargados de la Comisión de policía, á quienes toca imponer aquella.

Art. $4 .{ }^{\circ}$. Queda prohibido poner en la calle las basuras del interior de las casas, pues que deberán ser reservadas para que se depositen en los carros á su paso por la calle respectiva.

Art. 5.․ Las casas de matanza de cerdos, las pailas y las fábricas de cualquiera clase, tendrán en el exterior un rótulo que indique el objeto á que se hallan destinadas.

Art. $6 .^{\circ}$. Las caballerías y corrales de hoteles, mesones y cuarteles, deberán conservarse en perfecto estado de limpieza, teniendo á ellos libre acceso la policía para cerciorarse del cumplimiento de esta disposición.

Art. $7 .^{\circ}$. La comisión de instrucción primaria vigilará que las escuelas particulares estén en locales convenientes, atenta la concurrencia de alumnos, debiendo dar parte al Ayuntamiento de todo aquello que en su concepto sea contrario á la higiene, a fin de que dicte las medidas que sean oportunas según las circunstancias del caso.

Art. $8 .^{\circ}$. Solo en las Boticas, y mediante fórmula de facultativo, puede hacerse la venta de la planta Cannavis Indica conocida con el nombre de Marihuana.

Art. 9.․ No se depositarán los cadáveres en los templos para la celebración de actos religiosos ó con otro motivo, sin previo permiso del Presidente del Ayuntamiento, quien podrá concederlo sin que se le acredite con la constancia de dos facultativos que la presencia del cadáver en la iglesia no puede ocasionar ningún mal a los concurrentes á la misma.»11

Para la década de 1890, las obras de desecación de pantanos aún se encontraban en proceso, pero «el cumplimiento del Bando de Policía había dados buenos resultados, las fachadas de las casas de Morelia se pintaron y asearon, dándole a la ciudad un aspecto de alegría y se contribuía al mejoramiento de la higiene pública»12.

Durante la última década del porfiriato, el estado sanitario de la ciudad de Morelia había mejorado en muchos aspectos, y que no hubiese epidemia alguna representaba un gran trabajo en este ramo. La higiene y la salubridad de Morelia estaban vinculadas directamente con la limpieza; se tenía claro que si había un método de mejorar las condiciones sanitarias de la ciudad sería cumpliendo con el Bando de Policía, 
que se enfocaba principalmente en el aseo y la limpieza ${ }^{13}$.

En el número del Periódico Oficial del Gobierno del Estado correspondiente al 25 de julio de 1895 encontramos la siguiente noticia, con la cual podemos darnos cuenta de lo importantes que eran el aseo y la limpieza de las calles: «En el Ayuntamiento de la ciudad de México se discute actualmente la disposición que sigue: El barrido y regado se hará en las primeras horas de la mañana y entre una y dos de la tarde, y para que el aseo sea más completo, luego se barrerá fuertemente, se recogerá la basura y se volverá a regar, procurando no hacer charcos, para evitar los lodazales que suelen formarse. A los vecinos que no cumplan con esta disposición se les impondrá una multa de cinco á cincuenta pesos ${ }^{14}$.

Además de la limpieza de la ciudad, también había tomado importancia el aseo personal. En la década de 1880 ya se habían inaugurado algunos baños públicos en la ciudad, pero este no había sido un método muy eficaz, ya que gran parte de la población no contaba con el conocimiento sobre el beneficio de bañarse: «hay personas que tienen horror al baño, descuidando del aseo de la piel por este medio, y hombres hay que al paso que atienden con toda minuciosidad la limpieza de un caballo ó de otro animal doméstico, se olvidan de su propia piel, considerando muy eficiente el baño que recibieron con el agua del bautismo y no se lavan la cara sino el domingo ya que no se contenten con la simple ablución del remojo de la barba cuando se afeitan ${ }^{15}$.

Una forma de acercar a las personas hacia esta práctica del baño fue por medio de la prensa. Se hacían señalizaciones sobre la importancia de asear el cuerpo y sobre los beneficios que traía desarrollar esta práctica constantemente; un aspecto que nos parece interesante es sobre los tipos de baños, los de agua fría y los de agua caliente ${ }^{16}$.

En los últimos años del siglo XIX, para mejorar la salubridad de la ciudad se comenzaron a realizar estudios con el fin de implementar un buen sistema de conducción de aguas potables y atarjeas, por lo que se nombró una comisión para realizar dichas tareas ${ }^{17}$. Para el año 1901, los agentes de policía sanitaria continuaban funcionando como los guardianes de la higiene pública y su tarea principal continuaba siendo vigilar las disposiciones sanitarias ${ }^{18}$.

Entre los años 1878 y 1901, en la ciudad de Morelia se incrementó la mortalidad por varias enfermedades infecciosas, entre ellas el cólera, la viruela, el tifo, la tuberculosis y el paludismo. Las autoridades en turno se vieron obligadas a mantener al margen estas enfermedades echando mano de los preceptos higiénicos y las vacunas. Esto representó una ardua tarea para los higienistas y para el gobierno, ya que varios obstáculos dificultaban esta labor, entre ellos el desconocimiento sobre el origen y la transmisión de las enfermedades por muchos, los insuficientes conocimientos sobre la higiene y sus bondades, la resistencia de la población hacia la implementación de las vacunas, las extenuantes jornadas laborales y la mala alimentación de los trabajadores, y las paupérrimas condiciones higiénicas en las que vivían las grandes masas $^{19}$.

En el año 1891 se creó el Código Sanitario de los Estados Unidos Mexicanos, que en el año 1894 fue reformado. En ambas versiones apareció un capítulo dedicado a las enfermedades infecciosas y contagiosas, entre las cuales se mencionaban el cólera, el tifo, la fiebre tifoidea, el sarampión y la escarlatina. En el año 1895 se creó el Código Sanitario del Estado de Michoacán, que tenía como base el Código de la Federación. El dirigido a Michoacán, al igual que otros, contaba con un capítulo dedicado a las enfermedades infecciosas y contagiosas, y desarrollaba como temas el aislamiento, la desinfección, normas para las honras fúnebres y para la atención de enfermos en lugares de aglomeración, se mencionaba que era obligatoria la vacunación y debía hacerse el registro de las mujeres que ejercían la prostitución'.

Establecido el código sanitario y ya en funcionamiento para el año 1896, la salubridad del Estado de Michoacán, y en específico de la capital, pintaba un futuro prometedor, y se estableció un cuerpo de policía sanitaria que se encargaría de la inspección sobre la práctica de la higiene en la ciudad. El cuerpo mencionado se componía de cuatro agentes y tenían a su cargo cuidar de que las basuras, entendidas como «inmundas corrupciones», no envenenaran la atmósfera y no perjudicaran la salud de los vecindarios. Los agentes, que estaban bajo la dirección de un grupo de personas de ciencia, tenían también la tarea de educar a las familias sobre la aplicación de los preceptos de higiene privada y pública, y sobre no consumir bebidas y comestibles adulterados ${ }^{20}$.

\section{Conclusiones}

Durante el porfiriato se implementaron medidas para mejorar la higiene pública y privada en la ciudad de Morelia. Se priorizaron tareas como la desecación de los pantanos y la limpieza de la ciudad y del Río 
Grande. Se contó con reformas en el Bando de Policía que resultaron eficaces gracias a la estricta vigilancia por este cuerpo del cumplimiento de las medidas de higiene pública. Y se implementó el Código Sanitario del Estado de Michoacán, basado en el homónimo de la Federación, que normó actividades como el aislamiento, la desinfección, la aplicación obligatoria de vacunas y el registro de las mujeres que ejercían la prostitución.

\section{Conflicto de intereses}

Los autores declaran no tener conflictos de intereses.

\section{Responsabilidades éticas}

Protección de personas y animales. Los autores declaran que para esta investigación no se han realizado experimentos en seres humanos ni en animales.

Confidencialidad de los datos. Los autores declaran que en este artículo no aparecen datos de pacientes.

Derecho a la privacidad y consentimiento informado. Los autores declaran que en este artículo no aparecen datos de pacientes.

\section{Bibliografía}

1. Zavala-Ramírez MC. El arte de conservar la salud en el porfiriato. Higiene pública y prostitución en Morelia. Universidad Michoacana de San Nicolás de Hidalgo, Morelia; 2010. p. 21.

2. Tenorio-Trillo M. Artilugio de la Nación Moderna. México: Fondo de Cultura Económica; 1998. p. 200.
3. Memoria presentada por el ciudadano General de División Manuel González al ejecutivo de la unión, al Estado de Michoacán y a la legislatura del mismo sobre el uso de las facultades discrecionales que le fueron concedidas para reorganizar política y administrativamente dicho Estado. Morelia, julio de 1877. p. 10.

4. Periódico Oficial del gobierno del Estado de Michoacán de Ocampo. Sección Oficial, Gobierno Federal, Año III, Núm. 130, Morelia, 5 de marzo de 1880. p. 1.

5. Periódico Oficial del gobierno del Estado de Michoacán de Ocampo. Sección Oficial, Gobierno Federal, Año IV, Núm. 255, Morelia, 28 de mayo de 1881. p. 1.

6. Periódico Oficial del gobierno del Estado de Michoacán de Ocampo. Sección Oficial, Poder Legislativo, Año VIII, Núm. 353, Morelia, 20 de mayo de 1882. p. 3.

7. Gaceta Oficial del gobierno del Estado libre y soberano de Michoacán. Baños del Gran Hotel Oseguera, Tomo 1, Núm. 90, Morelia, 25 de julio de 1886. p. 2

8. Gaceta Oficial del gobierno del Estado libre y soberano de Michoacán. Nuevo panteón, Tomo I, Núm. 9, Morelia, 18 de octubre de 1885. p. 2.

9. Gaceta Oficial del gobierno del Estado libre y soberano de Michoacán. Pantanos, Tomo I, Núm. 39, Morelia, 31 de enero de 1886. p. 3.

10. Gaceta Oficial del gobierno del Estado libre y soberano de Michoacán. Desecación de los pantanos, Año II, Núm. 158, Morelia, 3 de abril de 1887. p. 1.

11. Gaceta Oficial del gobierno del Estado libre y soberano de Michoacán, Año II, Núm. 161, Morelia, 17 de abril de 1887. p. 1-2.

12. Gaceta Oficial del gobierno del Estado libre y soberano de Michoacán, Año V, Núm. 433, Morelia, 22 de diciembre de 1889. p. 3.

13. Periódico Oficial del gobierno del Estado de Michoacán de Ocampo, Tomo II, Núm. 4, Morelia, 1 de noviembre de 1894. p. 7.

14. Periódico Oficial del gobierno del Estado de Michoacán de Ocampo. Bandos de Policía, Tomo III, Núm. 59, Morelia, Jueves 25 de julio de 1895. p. 6.

15. Periódico Oficial del gobierno del Estado de Michoacán de Ocampo. Los baños y su origen, Tomo I, Núm. 69, Morelia, Domingo 27 de agosto de 1893. p. 5.

16. Periódico Oficial del gobierno del Estado de Michoacán de Ocampo. Higiene del Baño, Tomo I, Núm. 39, Morelia, Domingo 14 de mayo de 1893. p. 6.

17. Periódico Oficial del gobierno del Estado de Michoacán de Ocampo, Tomo VIII, Núm. 74, Morelia, 16 de septiembre de 1900. p. 1.

18. Periódico Oficial del gobierno del Estado de Michoacán de Ocampo, Tomo IX, Núm. 35, Morelia, Jueves 2 de mayo de 1901. p. 1.

19. Rivera-Tapia JA. La situación de la salud pública en México (1870-1960). Rev Hosp Gral Dr. M Gea González. 2003;6(1):40-4.

20. La libertad, Policía Sanitaria en Morelia, Año $5 .^{\circ}$, tomo $5 .^{\circ}$, Núm. 39, Morelia, Martes 28 de septiembre de 1897, p. 1; Periódico Oficial del gobierno del Estado de Michoacán de Ocampo, Tomo VI, Núm. 44, Morelia, Jueves 2 de junio de 1898. p. 2. 